\title{
A Novel Agricultural Commodity Price Forecasting Model Based on Fuzzy Information Granulation and MEA-SVM Model
}

\author{
Yongli Zhang ${ }^{1}{ }^{1}$ and Sanggyun $\mathrm{Na}^{2}$ \\ ${ }^{1}$ School of Management Science and Engineering, Hebei GEO University, Shijiazhuang, Hebei Province, China \\ ${ }^{2}$ College of Business Administration, Wonkwang University, Iksan, Jeonbuk, Republic of Korea \\ Correspondence should be addressed to Yongli Zhang; zhangyongli086@163.com
}

Received 4 September 2018; Revised 27 October 2018; Accepted 29 October 2018; Published 11 November 2018

Academic Editor: Guang-Yong Sun

Copyright (C) 2018 Yongli Zhang and Sanggyun Na. This is an open access article distributed under the Creative Commons Attribution License, which permits unrestricted use, distribution, and reproduction in any medium, provided the original work is properly cited.

\begin{abstract}
Accurately predicting the price of agricultural commodity is very important for evading market risk, increasing agricultural income, and accomplishing government macroeconomic regulation. With the price index predictions of 6 commodities of Food and Agriculture Organization of the United Nations (FAO) as examples, this paper proposed a novel agricultural commodity price forecasting model which combined the fuzzy information granulation, mind evolutionary algorithm (MEA), and support vector machine (SVM). Firstly, the time series data of agricultural commodity price index was transformed into fuzzy information granulation particles made up of $L o w, R$, and $U p$, which represented the trend and magnitude of price movement. Secondly, MEA algorithm was employed to seek the optimal parameters $c$ and $g$ for SVM to establish the MEA-SVM model. Finally, FOA price index fluctuation range and change trend in the future were predicted by the MEA-SVM model. The empirical analysis showed that the MEA-SVM model was effective and had higher prediction accuracy and faster calculation speed in the forecasting of agricultural commodity price.
\end{abstract}

\section{Introduction}

The scientific and effective forecasting method is conducive to correctly guide agricultural production, realize the balance of supply and demand of agricultural commodities, increase peasants' income, and provide decision-making basis for the government to adjust agricultural economic structure and implement targeted macro-control [1-3].

Nowadays, the agricultural price forecasting methods can be divided into two categories: qualitative forecasting methods and quantitative forecasting methods. As a supplement to other agricultural commodity price forecasting methods, qualitative forecasting methods generally do not occupy the mainstream position, with low accuracy and high subjectivity. According to the sequence of occurrence time, the quantitative prediction methods can be divided into econometric method, time series analysis method, and intelligent prediction method. The econometric method finds the supporting economic theory according to the research problem, then puts forward the hypothesis, and establishes the econometric model to verify the hypothesis [4-9]. However, most empirical studies do not prove that the prediction effect of the classic econometric model is better than that of the time series analysis method [10-12]. Therefore, the time series analysis method gradually replaced the econometric method in the 1990s. Due to the complexity and difficulty of price prediction of agricultural commodities, price fluctuations always show the characteristics of repeated ups and downs, instability, benign or vicious circle, etc. The advantages of self-adaptation, self-learning, and self-organization possessed by the intelligent prediction method can well adapt to the characteristics of market price fluctuations of agricultural commodities. Therefore, in recent years, the intelligent prediction method has been increasingly applied to the prediction of agricultural price [13-17]. Intelligent prediction methods usually include artificial neural network, chaos theory, entropy analysis, extreme learning machines, radial basis function, and support vector regression.

The deficiency of the above prediction methods is to use a single model to predict the time series data of agricultural 
commodities. Meanwhile, most forecasting methods provide a point estimation rather than a value range. In the real world, peasants and governments pay more attention to the fluctuation range and trend of agricultural prices. Our study firstly transforms time series data of prices index into fuzzy information granulation particles made up of Low, $R$, and $U p$, which represent the trend and magnitude of price movements, then employs a hybrid method that combines the support vector machine (SVM) with mind evolutionary algorithm (MEA) to predict the fuzzy information granulation particles Low, $R$, and $U p$, and finally captures the fluctuation range and change trend exhibited in agricultural commodity futures prices, contributing to existing studies and filling the research gap.

Besides, if the SVM model wants to achieve a relatively ideal regression effect, it must set and adjust relevant parameters; the most important parameters are the penalty parameter $c$ and the kernel function parameter $g$. Previous studies applied grid search method (GSM) to seek the optimal parameters $c$ and $g$ of SVM [18-21]. Although GSM can obtain the global optimal solution, that is, the optimal regression accuracy, it is usually conducted within the specified range. Once the search range is expanded, the algorithm training time will be very long [22-24]. To further improve the optimization performance and overcome the deficiencies of traditional algorithms, the Chinese scholar Sun ChengYi et al. raised the mind evolutionary algorithm (MEA) in 1998 [25]. MEA is an evolutionary algorithm simulating the progress of human mind and has the positive and negative feedback mechanism, wherein the positive feedback mechanism improves toward being more beneficial to the population survival to consolidate and develop the evolution achievement. The negative feedback mechanism prevents the algorithm premature to avoid that the algorithm is caught in local optimal solution. The structural parallelism of MEA guarantees the high search efficiency of the algorithm, overcomes the defects of traditional evolutionary algorithms such as time-consuming computation and premature, and also has extreme robustness on interference [26, 27]. Although the MEA has been applied in some technical fields [28-30], there are little credible researches employing MEA to optimize the parameters of the SVM model. This study utilized MEA to optimize the penalty parameter $c$ and the kernel function parameter $g$ of SVM and established the novel MEA-SVM model, which provides a methodological perspective for the agricultural price forecasting, extending the prior researches.

Taking the price indexes of Food and Agriculture Organization of the United Nations (FAO) as examples, this study took 6 consecutive trading months as one group, adopted fuzzy information granulation theory to map the time series data into 3 fuzzy information particles with low boundary value Low, median value $R$, and high boundary value $U p$, then applied MEA to search for the optimal parameters $c$ and $g$ of the SVM model, established the MEA-SVM model, and finally employed the hybrid MEA-SVM model to predict the lowest, average, and highest prices of the FAO price index in the future period, so as to obtain the future fluctuation interval and variation trend of the FAO price index.
The rest of this article is organized as follows. The fuzzy information granulation theory, SVM model, and MEA are introduced, and the MEA-SVM model is constructed in Section 2. The empirical analysis is given, six agricultural commodity price indexes are predicted, and the performance of MEA-SVM model and GSM-SVM model is compared in Section 3. At last, conclusions are summarized and future research is suggested in Section 4.

\section{Model Construction}

2.1. Fuzzy Information Granulation Theory. The concept of information granulation was first proposed by American scholar Zadeh [31]. Information granules are collections of objects, which are combined together due to functional similarity, proximity, indivisibility, and functionality. Granulation calculation is a new computing paradigm covering granulation theory, technology, method, and tools. It integrates computing theory, entropy space theory, rough set theory, and interval calculation and is one of the hot spots in the field of artificial intelligence and soft computing science.

Information granulation methods mainly include rough set information granulation theory, fuzzy set information granulation theory, and entropy space information granulation theory. Wherein, the information grains represented by fuzzy sets are called fuzzy information grains. When the fuzzy set method granulates the time series data, it mainly includes two steps: window division and fuzzy processing. The original time sequence is divided into several subsequences, which is called window division; the divided subwindows are fuzzified to generate fuzzy sets, which is called fuzzification. The combination of the two treatments is fuzzy information granulation, also known as $f$ granulation. Assuming that the time series data $X$ is regarded as one window, the task of fuzzy processing is to establish a fuzzy information particle $P$, which can replace the original window data. In other words, the fuzzy concept $G$ (fuzzy set with $X$ as the theoretical domain) is determined reasonably so as to describe $X[32,33]$.

Therefore, the essence of fuzzy process is the process of determining the membership function $A$ of the fuzzy concept $G$. The general membership functions of fuzzy particles include triangle, parabola, Gaussian, and trapezoid. In this study, triangular fuzzy particles are adopted and their membership functions are shown as follows.

$$
A(x, a, m, b)= \begin{cases}0, & x<a ; \\ \frac{x-a}{m-a}, & a \leq x \leq m ; \\ \frac{b-x}{b-m}, & m \leq x \leq b ; \\ 0, & x>b\end{cases}
$$

In the formula, the original time series data is represented by $x$, and the minimum, average, and maximum values of the original data are, respectively, described by fuzzy particle parameters $a, b$, and $m$.

2.2. Support Vector Machine. The support vector machine (SVM) model was first proposed by Vapnik in 1995 [34]. 
It can be used for both pattern classification and nonlinear regression analysis. SVM model is a supervised learning algorithm based on the structure risk minimum principle and statistical Vapnik-Chervonenkis dimension theory. The main idea of SVM model is to transform the nonlinear classification problem into a multidimensional linear convex quadratic programming problem through kernel function mapping and establishment of classification higher-dimensional decision surface. The complexity of SVM algorithm is independent from the sample dimension. It has the advantages of universality, robustness, validity, simple calculation, perfect theory, etc. The obtained solution is unique and globally optimal, which avoids the problem that the neural network method falls into local extreme value [35-37].

In the case of linear separability, the core of SVM algorithm is to find the optimal classification decision plane. It is assumed that the sample data set of linear separable training is $T$.

$$
T=\left\{\left(x_{1}, y_{1}\right),\left(x_{2}, y_{2}\right), \ldots,\left(x_{l}, y_{l}\right)\right\} \in(x \times y)^{l}
$$

In the formula, $x_{i} \in R^{n}, y_{i} \in\{-1,1\}, i=1, \ldots, l$.

The construction of the secondary planning problem is expressed as follows.

$$
\begin{array}{ll}
\min _{\omega, b} & \frac{1}{2}\|\omega\|^{2}, \\
\text { s.t. } & y_{i}\left(\left(\omega \bullet x_{i}\right)+b\right) \geq 1, \quad i=1, \ldots, l
\end{array}
$$

The classification surface determined by the optimal solution is expressed as follows.

$$
\left(\omega^{*} \cdot x\right)+b^{*}=0
$$

The decision function is constructed as follows.

$$
f(x)=\operatorname{sgn}\left(\left(\omega^{*} \cdot x\right)+b^{*}\right)
$$

SVM model is characterized by sparsity and uses a few support vectors to represent decision functions. In order to guarantee this important characteristic, when SVM algorithm is applied to solve nonlinear regression problems, the loss function needs to be introduced. Taking the standard insensitive loss function $\varepsilon$ as an example, the form of the $\varepsilon$ SVM model is as follows.

$$
\begin{array}{ll}
\min & \frac{1}{2}\|\omega\|^{2}+C \sum_{i=1}^{m}\left(\xi_{i}+\xi_{i}^{*}\right) \\
\text { s.t. } & \left\{\begin{array}{l}
y_{i}-\omega \cdot x_{i}-b \leq \varepsilon+\xi_{i} \\
\omega \cdot x_{i}+b-y_{i} \leq \varepsilon+\xi_{i}^{*} \\
\xi_{i}, \xi_{i}^{*} \geq 0
\end{array}\right.
\end{array}
$$

In the formula, $\xi_{i}$ and $\xi_{i}^{*}$ are relaxation variables and represent the model error requirements; $C$ is the penalty parameter, which is used to measure the degree of matching between empirical risk and confidence range.
2.3. Mind Evolutionary Algorithm. Mind Evolutionary Algorithm (MEA) is a kind of evolutionary algorithm simulating the progress of human mind, proposed for the defects of genetic algorithm (GA). The MEA uses two types of operation: similar taxis and dissimilation, and it uses population optimizing instead of individual optimizing. The similar taxis operation is a process in which the individual competes to be a winner, which happens within the scope of subpopulation. The dissimilation operation is the process in which a subpopulation battles to be a winner and continuously explores the new point of solution space, which happens within the whole solution space. In the course of the algorithm running, the similar taxis and dissimilation operation is executed repeatedly until the terminating condition is satisfied [25].

Compared with GA, the MEA has the following advantages: The crossover and mutation operations of GA generate not only superior genes, but also inferior destructive genes, and those operations have duality, but the MEA uses the similar taxis and dissimilation operations, which amend the defects of GA. The similar taxis and dissimilation operations of MEA are coordinated mutually but also are independent mutually, and any improvement on any aspect will raise the algorithm's prediction accuracy. The similar taxis and dissimilation operations have parallelism on structure, which boosts the algorithm's search efficiency and computation speed. The MEA divides the populations into superior subpopulations and temporary subpopulations, which can memorize evolutionary information more than one generation $[38,39]$.

The MEA follows some basic concepts of the GA such as "population," "individual," and "environment", but, meanwhile, it also adds some new concepts.

2.3.1. Population and Subpopulation. The MEA is a kind of learning method making optimization through iteration, and all individuals in every generation of the evolutionary process gather into one population. A population is divided into several subpopulations. The subpopulation contains two classes: superior subpopulation and temporary subpopulation. The superior subpopulation records the information of the winners in the global competition, and the temporary subpopulation documents the process of the global competition.

2.3.2. Billboard. The billboard is equivalent to an information platform, which provides chances of information communication between the individuals or the subpopulations. The billboard records three types of useful information: the serial number of individual or subpopulation, the action, and the score. By utilizing the serial number of individual or subpopulation, it is convenient to distinguish different individuals or subpopulations; the description of action varies from different research fields, and since this article is researching the problem of parameter optimization, and the action is used to record the exact position of the individual and subpopulation, the score is the evaluation of environment on the individual action, and in the optimization process by utilizing the MEA, it can rapidly find out the optimized individuals 
and populations only if the scores of every individual and subpopulation are recorded all the time. The individuals in the subpopulation post up their own information on the local billboard, and the information of each subpopulation is posted up on the global billboard.

2.3.3. Similar Taxis. Within the scope of subpopulation, the process that an individual competes to be a winner is called similar taxis. In the process of a subpopulation's similar taxis, if a new winner cannot be generated, it means that such subpopulation has matured. When a subpopulation matures, the similar taxis process of such subpopulation comes to an end. The period of a subpopulation from its birth to maturity is called the lifetime.

2.3.4. Dissimilation. In the whole solution space, each subpopulation competes to be a winner and continuously explores new solution space point; this process is known as dissimilation. The dissimilation has two definitions: each subpopulation makes global competition, and if the score of a temporary subpopulation is higher than the score of a specific matured superior subpopulation, such superior subpopulation will be replaced by the winning temporary subpopulation, and the individuals of the original superior subpopulation will be released; if the score of a matured temporary subpopulation is lower than the score of any superior subpopulation, such temporary subpopulation will be abandoned, and the individuals therein will be released; the released individuals will research and form new temporary subpopulation.

2.3.5. MEA-SVM Model. In this study, the prediction accuracy of training set was taken as the fitness function, and MEA was applied to seek the best penalty parameter $c$ and kernel function parameter $g$ of SVM model. The overall process of the algorithm is shown in Figure 1.

The main forecasting process of agricultural commodity price based on fuzzy information granulation and MEA-SVM model is described below.

Step 1. Extract time series data of agricultural commodity price index.

Step 2. The triangular fuzzy information granulation theory is applied to process the time series data of the original agricultural commodity price index, and three fuzzy information particles representing the lowest, average, and highest prices of the original agricultural commodity price are obtained.

Step 3. MEA was adopted to search for the optimal parameters $c$ and $g$ of SVM model.

Step 4. The hybrid MEA-SVM model is applied to predict the agricultural commodity price index for 1-6 and 7-12 months in the future.

Step 5. Verify and evaluate the predicted results.
2.4. Model Assessment Criteria. In this study, mean squared error (MSE), squared correlation coefficient $\left(R^{2}\right)$, and computation time (second) are employed to measure the prediction accuracy and computation speed of MEA-SVM model. The calculation formulas of MSE and $R^{2}$ are as follows:

$$
\begin{aligned}
& \text { MSE }=\frac{1}{n} \sum_{i=1}^{n}\left(f\left(x_{i}\right)-y_{i}\right)^{2} \\
& R^{2} \\
& =\frac{\left(n \sum_{i=1}^{n} f\left(x_{i}\right) y_{i}-\sum_{i=1}^{n} f\left(x_{i}\right) \sum_{i=1}^{n} y_{i}\right)^{2}}{\left(n \sum_{i=1}^{n} f\left(x_{i}\right)^{2}-\left(\sum_{i=1}^{n} f\left(x_{i}\right)\right)^{2}\left(n \sum_{i=1}^{n} y_{i}^{2}-\left(\sum_{i=1}^{n} y_{i}\right)^{2}\right)\right.}
\end{aligned}
$$

\section{Empirical Analysis}

3.1. Data Sources. This study selected the time series data of FAO price index (http://www.fao.org/home/en/) as the empirical research object. The FAO food price index is a measure of the monthly change in international prices of a basket of food commodities. It consists of the average of six commodity group price indexes (Food Price Index, Cereal Price Index, Vegetable Oil Price Index, Meat Price Index, Dairy Price Index, and Sugar Price Index). The MEASVM model takes the time series data of price index of six commodities from January 1990 to June 2017 as training data and the time series data from July 2017 to June 2018 as testing data. As an example, the FAO food price index from January 1990 to June 2017 is shown in Figure 2. Based on this, the highest price, lowest price, average price, and the variation trend of the FAO food price index from July 2017 to June 2018 will be predicted.

3.2. Fuzzy Information Granulation. The time series data of 330 months from January 1990 to July 2017 were regarded as the training set and every 6 months was treated as one information granulation window. Finally, the FAO food price index is transformed into 55 fuzzy information particles, each containing 3 parameters of Low, $R$, and $U p$, as shown in Figure 3. For one single fuzzy information particle, parameters Low, $R$, and Up describe the minimum, mean, and maximum values of original data changes, respectively.

3.3. Regression Prediction of Granulation Data. The hybrid MEA-SVM model was employed to predict the Low, $R$, and Up. Firstly, the granulation data was preprocessed, then MEA was applied to search the best penalty parameter $c$ and kernel function parameter $g$ for establishing the MEA-SVM model. Finally, the MEA-SVM model was used for training and prediction. For example, the prediction process of parameter Low is as follows.

3.3.1. Data Preprocessing. In this study, the granulation data of Low was normalized and data normalization range was $[100,500]$, as shown in Figure 4.

3.3.2. Searching for the Optimal Parameters $c$ and $g$. In this study, MEA was adopted to search for the best parameters $c$ 


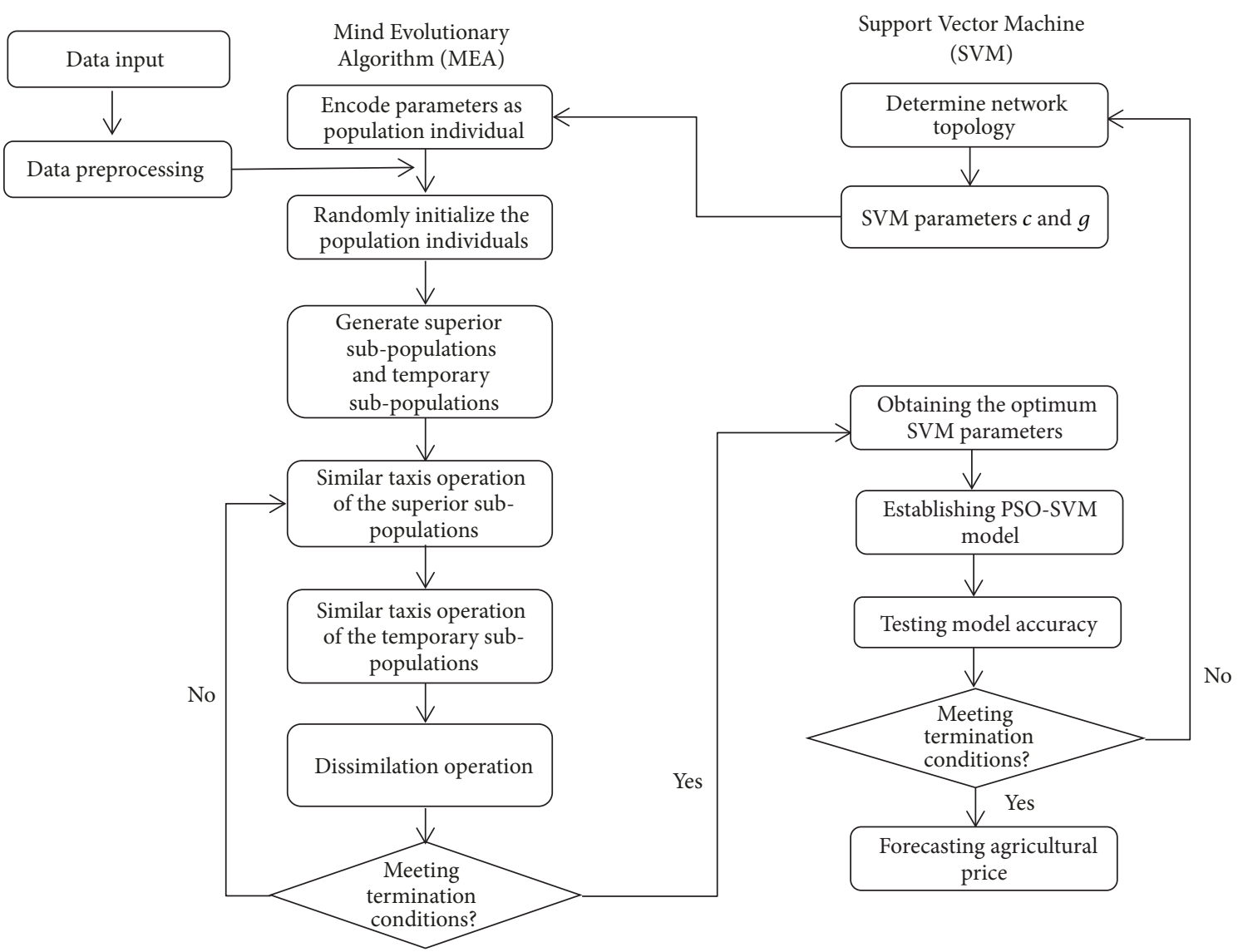

FIgURE 1: The process of SVM parameters optimized by MEA.

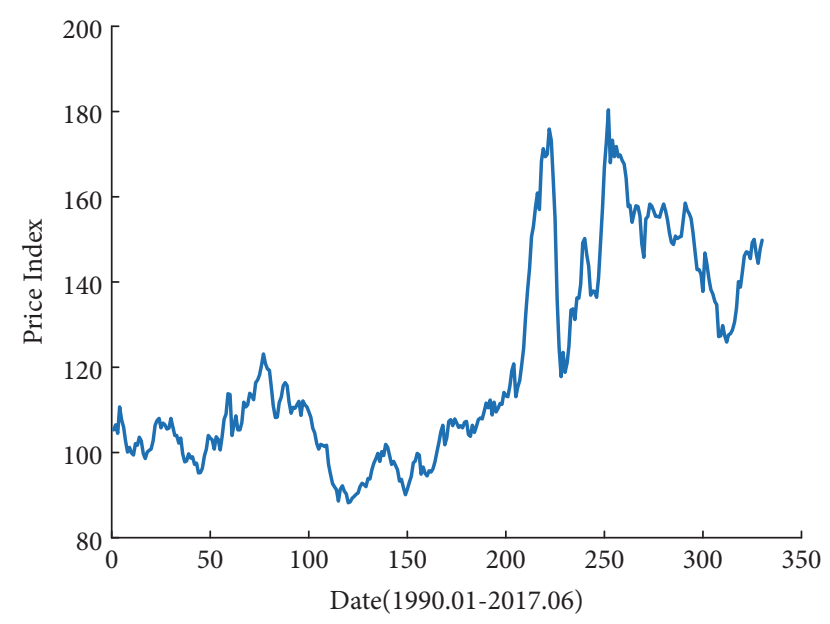

FIgURE 2: The FAO food price index (1990.01 2017.06).

and $g$ of SVM model. The population size was set to 200 , the numbers of superior population and temporary population size were set to 5 , subpopulation size was set to 20 , and evolution generations are set to 200 . The minimum-value and maximum-value of $c$ were 500 and 10 . The minimum-value and maximum-value of $g$ were 0.01 and 1 . The minimumvalue and maximum-value of $p$ were 0.01 and 100 .

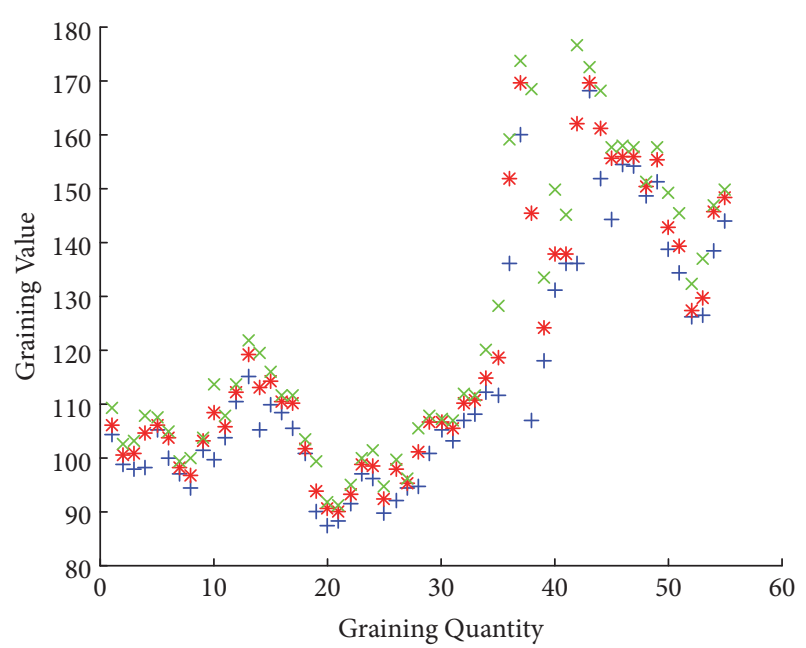

$$
\begin{aligned}
& + \text { Low } \\
& * \mathrm{R}
\end{aligned}
$$

FIGURE 3: Visualization diagram of fuzzy information granulation.

The optimal parameter search processes of MEA for granulated data Low were shown in Figures 5 and 6.

In order to verify the superiority of MEA, GSM was also employed to search the optimal parameters $c$ and $g$ of SVM 


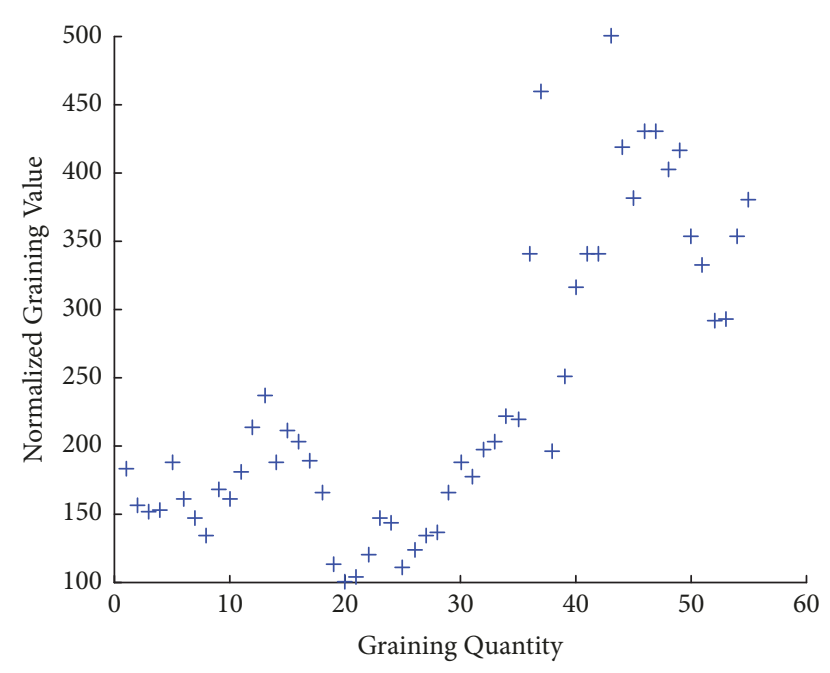

FIGURE 4: The normalization of the parameter Low.

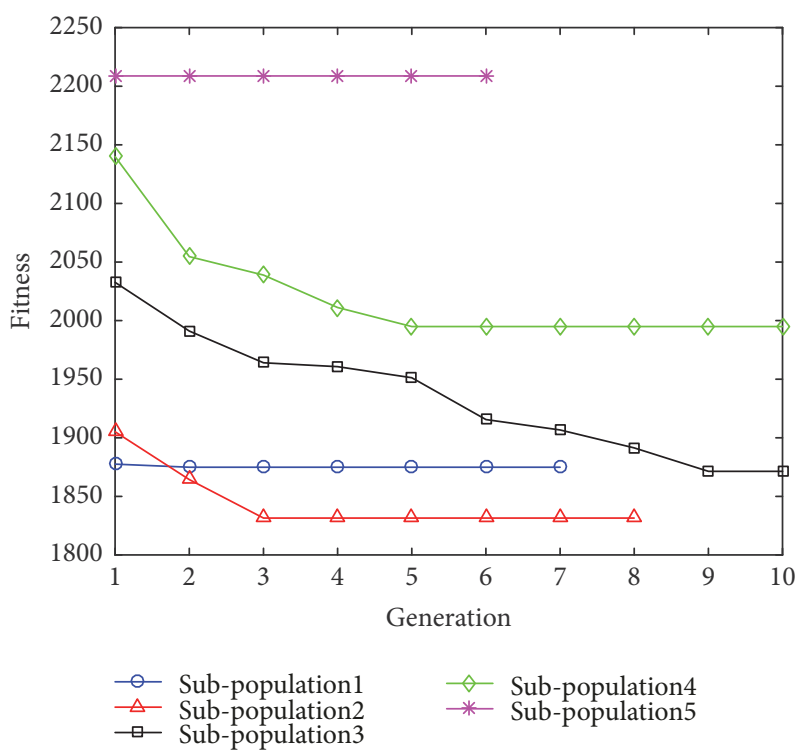

FIGURE 5: The convergence process of superior subpopulation for granulated data Low.

model on the basis of the same parameter settings. The GSM first looks for the optimal parameters roughly (Figure 7) and then delicately seeks for the optimal parameter after observing the cursory search results (Figure 8).

In Figures 7 and 8 , the $X$-axis represents the log base 2 of $c$, the $Y$-axis represents the log base 2 of $g$, the contour line represents the MSE corresponding to $c$ and $g$.

Finally, the optimal parameters obtained by the MEA and GSM for granulation data Low of six FAO price indexes are shown in Table 1.

3.3.3. Model Training. The optimal parameters $c$ and $g$ were obtained and then substituted into the SVM model to establish the MEA-SVM model. The lowest, average, and highest prices of FAO food price index from January 1990 to June 2017 were predicted by using the MEA-SVM model.

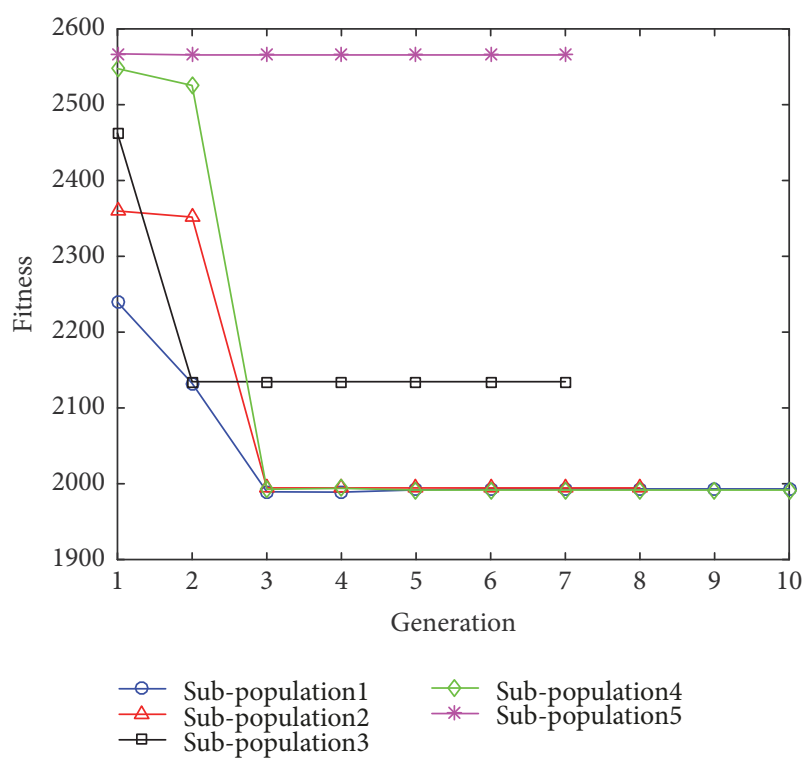

FIGURE 6: The convergence process of temporary subpopulation for granulated data Low.
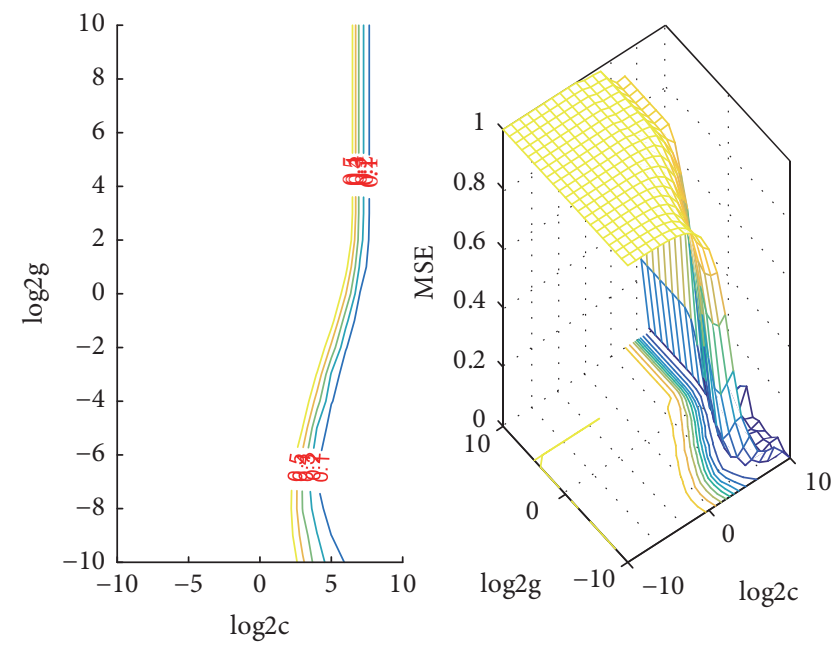

FIGURE 7: The cursory parameter searching results of GSM for granulated data Low.

The predicted values, actual values, and relative errors obtained by MEA-SVM for granulation data Low of FAO food price index were shown in Figures 9 and 10. Meanwhile, the performance of MEA-SVM in predicting granulation data Low is compared with GSM-SVM, as shown in Table 2.

In Table 2, the lower the MSE and the higher the $R^{2}$, the more accurate the model's prediction. Excluding oils price index, the comparison results of the other five agricultural commodity price indexes show that the prediction accuracy of the MEA is superior to the GSM. At the same time, the calculation speed is improved.

3.3.4. Model Prediction. The 6-month FAO price index of granulation data Low is regarded as one period, and the fluctuation range and change trend of the 12-month FAO food price index in the next two periods are predicted. 
TABLE 1: The optimal parameters obtained by the MEA and GSM.

\begin{tabular}{lcccc}
\hline Price Index & \multicolumn{2}{c}{ MEA } & G & GSM \\
\hline Food Price Index & $c$ & 0.0692 & 256.0000 & 0.0313 \\
Meat Price Index & 291.8520 & 0.0292 & 64.0000 & 0.0313 \\
Dairy Price Index & 155.9980 & 0.2151 & 256.0000 & 0.1768 \\
Cereals Price Index & 306.3320 & 0.0634 & 256.0000 & 0.0625 \\
Oils Price Index & 287.6840 & 0.1410 & 181.0190 & 0.2500 \\
Sugar Price Index & 115.0080 & 0.4890 & 181.0190 & 0.2500 \\
\hline
\end{tabular}

TABLE 2: The prediction of food price index for granulation data Low.

\begin{tabular}{|c|c|c|c|c|c|c|}
\hline \multirow{2}{*}{ Price Index } & \multicolumn{3}{|c|}{ MEA } & \multicolumn{3}{|c|}{ GSM } \\
\hline & MSE & $R^{2}$ & Time(s) & MSE & $R^{2}$ & Time(s) \\
\hline Food Price Index & 1199.1000 & 0.8970 & 14.7905 & 1523.8400 & 0.8687 & 17.2127 \\
\hline Meat Price Index & 1235.8600 & 0.8500 & 7.2805 & 1309.0300 & 0.8417 & 10.1516 \\
\hline Dairy Price Index & 471.6220 & 0.9436 & 10.0107 & 680.0180 & 0.9238 & 10.4906 \\
\hline Cereals Price Index & 1465.9800 & 0.8697 & 9.0348 & 1473.0900 & 0.8693 & 10.6664 \\
\hline Oils Price Index & 1611.9100 & 0.8012 & 10.4889 & 1090.5900 & 0.8765 & 11.1705 \\
\hline Sugar Price Index & 656.3550 & 0.9452 & 10.5295 & 1229.6400 & 0.8993 & 10.9678 \\
\hline
\end{tabular}

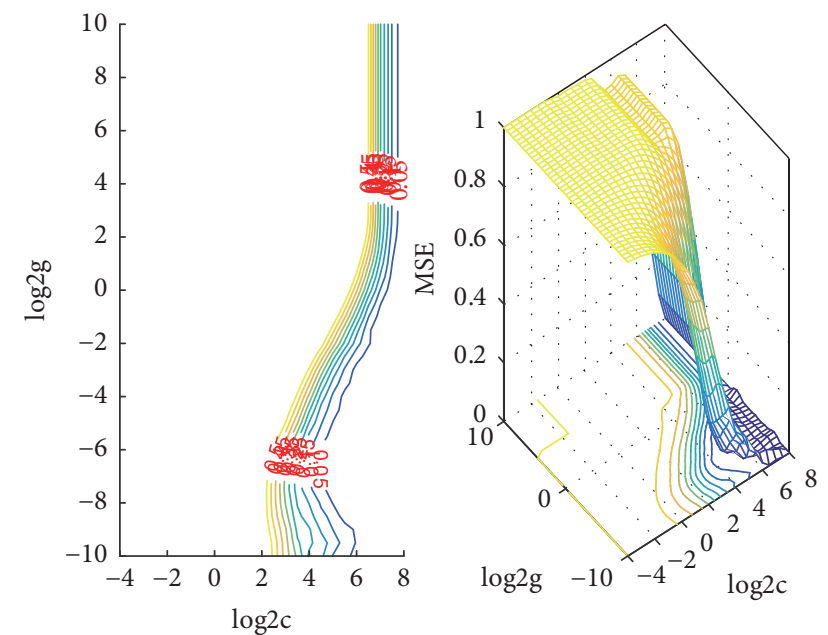

FIGURE 8: The delicate parameter searching results of GSM for granulated data Low.

By repeating the above data preprocessing, searching for the optimal parameters $c$ and $g$, model training and model prediction for the granulation data of $R$ and $U p$, the predicted values of 3 fuzzy particles Low, $R$, and $U p$ in the next two periods were finally obtained (Table 3 ).

3.4. Predictive Performance Evaluation. The actual price indexes of six commodities from July 2017 to June 2018 were compared with the predicted price indexes to test the prediction effect of fuzzy information granulation and MEA-SVM model (Tables 4 and 5). For instance, from July 2017 to December 2017, the highest price, average price, and lowest price of FAO food price index are 144.4461, 150.3121, and 152.9865 , respectively, and the corresponding predicted

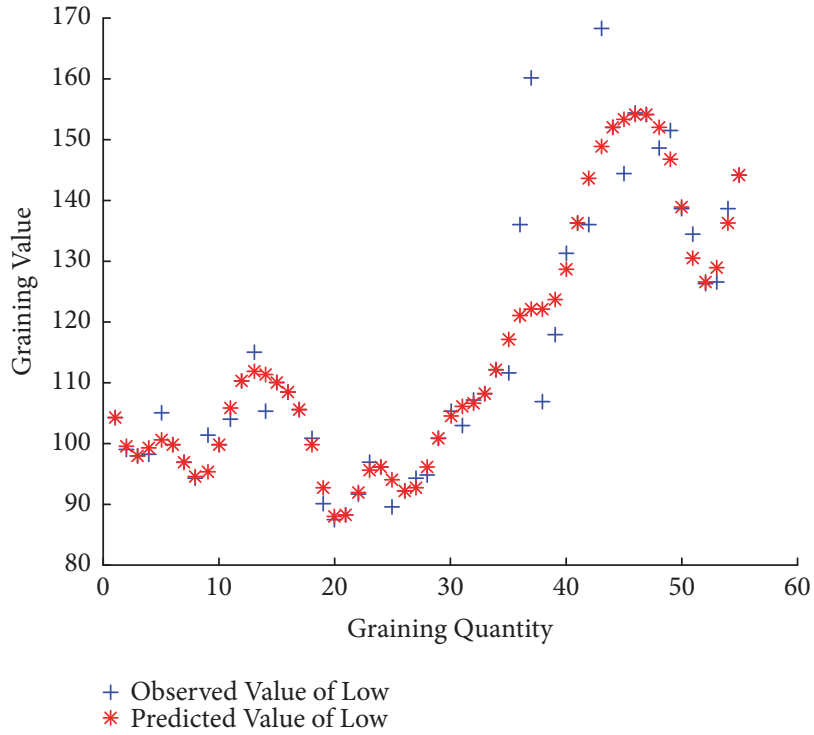

FIGURE 9: The comparison of observed and predicted values of granulation data Low.

values are $147.3770,145.7625$, and 143.4025 , with the relative prediction errors of $2.03 \%,-3.03 \%$, and $-6.26 \%$.

In terms of mean value $R$ of the price index, the predictions of cereals price index and meat price index are the most accurate; the dairy price index has the biggest forecasting error. For many FOA price indexes, the predicted values of granulation data Low, $R$, or Up are lower than the actual observed values, and there are risks of price underestimation. Overall, the prediction error of the second period is higher than that of the first period, so the further the prediction period is, the larger the prediction error is. The minimum and maximum values of the mean prediction relative errors 
TABLE 3: The prediction of FAO price indexes fluctuation range and change.

\begin{tabular}{|c|c|c|c|c|c|c|}
\hline \multirow{2}{*}{ Price Index } & \multicolumn{3}{|c|}{ 2017.07-2017.12 } & \multicolumn{3}{|c|}{ 2018.01-2018.06 } \\
\hline & Low & $R$ & Up & Low & $R$ & $U p$ \\
\hline Food Price Index & 147.3770 & 145.7625 & 143.4025 & 144.6949 & 136.3491 & 134.4704 \\
\hline Meat Price Index & 132.0103 & 145.1837 & 149.2589 & 130.0265 & 142.0818 & 141.1972 \\
\hline Dairy Price Index & 160.0152 & 142.1009 & 174.9390 & 142.9604 & 124.0123 & 163.0491 \\
\hline Cereals Price Index & 124.0533 & 129.9318 & 136.9791 & 122.4561 & 123.7921 & 132.2620 \\
\hline Oils Price Index & 129.8639 & 134.1359 & 149.2905 & 119.2376 & 124.1266 & 138.5303 \\
\hline Sugar Price Index & 139.3746 & 187.3576 & 200.8544 & 135.7445 & 166.7963 & 176.4463 \\
\hline
\end{tabular}

TABle 4: Predictive performance evaluation for FAO price indexes from July 2017 to December 2017.

\begin{tabular}{lccc}
\hline Price Index & $\begin{array}{c}\text { Actual Variation } \\
{[\text { Low, } R, U p]}\end{array}$ & $\begin{array}{c}\text { Predicted Variation } \\
{[\text { Low, } R, U p]}\end{array}$ & $\begin{array}{c}\text { Relative Error } \\
{[\text { Low, } R, \text { Up }]}\end{array}$ \\
\hline Food Price Index & {$[144.4461,150.3121,152.9865]$} & {$[147.3770,145.7625,143.4025]$} & {$[2.03 \%,-3.03 \%,-6.26 \%]$} \\
Meat Price Index & {$[144.9577,148.2275,150.0517]$} & {$[132.0103,145.1837,149.2589]$} & {$[-8.93 \%,-2.05 \%,-0.53 \%]$} \\
Dairy Price Index & {$[157.5603,179.7687,191.5383]$} & {$[160.0152,142.1009,174.939]$} & {$[1.56 \%,-20.95 \%,-8.67 \%]$} \\
Cereals Price Index & {$[129.7582,131.7769,138.5574]$} & {$[124.0533,129.9318,136.9791]$} & {$[-4.40 \%,-1.40 \%,-1.14 \%]$} \\
Oils Price Index & {$[137.0251,142.0297,147.0943]$} & {$[129.8639,134.1359,149.2905]$} & {$[-5.23 \%,-5.56 \%, 1.49 \%]$} \\
Sugar Price Index & {$[168.5479,174.9175,181.7145]$} & {$[139.3746,187.3576,200.8544]$} & {$[-17.31 \%, 7.11 \%, 10.53 \%]$} \\
\hline
\end{tabular}

TABLE 5: Predictive performance evaluation for FAO price indexes from January 2018 to June 2018.

\begin{tabular}{lccc}
\hline Price Index & $\begin{array}{c}\text { Actual Variation } \\
{[\text { Low }, R, U p]}\end{array}$ & $\begin{array}{c}\text { Predicted Variation } \\
{[\text { Low, } R, \text { Up }]}\end{array}$ & $\begin{array}{c}\text { Relative Error } \\
{[\text { Low, } R, \text { Up }]}\end{array}$ \\
\hline Food Price Index & {$[143.9263,147.6488,150.4290]$} & {$[144.6949,136.3491,134.4704]$} & {$[0.53 \%,-7.65 \%,-10.61 \%]$} \\
Meat Price Index & {$[143.1188,145.0220,146.0823]$} & {$[130.0265,142.0818,141.1972]$} & {$[-9.15 \%,-2.03 \%,-3.34 \%]$} \\
Dairy Price Index & {$[153.7400,171.0197,183.8850]$} & {$[142.9604,124.0123,163.0491]$} & {$[-7.01 \%,-27.49 \%,-11.33 \%]$} \\
Cereals Price Index & {$[133.8120,141.0704,147.4464]$} & {$[122.4561,123.7921,132.262]$} & {$[-8.49 \%,-12.25 \%,-10.30 \%]$} \\
Oils Price Index & {$[124.8099,132.3246,139.3903]$} & {$[119.2376,124.1266,138.5303]$} & {$[-4.46 \%,-6.20 \%,-0.62 \%]$} \\
Sugar Price Index & {$[149.7763,157.6108,170.8406]$} & {$[135.7445,166.7963,176.4463]$} & {$[-9.37 \%, 5.83 \%, 3.28 \%]$} \\
\hline
\end{tabular}

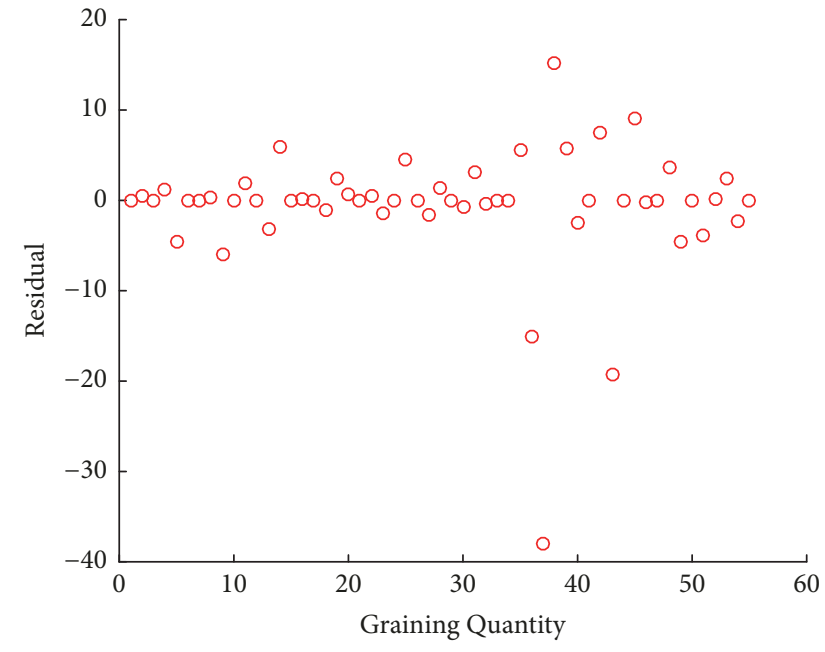

FIGURE 10: The prediction error of granulation data Low.

are $-1.40 \%$ and $-20.95 \%$ in the first period and $-2.03 \%$ and $-27.49 \%$ in the second period. The prediction of the future fluctuation range of FAO price index time series data by fuzzy information granulation and MEA-SVM model is generally accurate.

As for the forecast of price indexes change trend, the price of the second period is lower than that of the first period, which is consistent with the observation of FAO price indexes. The prediction of future change trend of FAO price index time series data by fuzzy information granulation and MEA-SVM model is correct.

\section{Conclusion and Discussion}

Many uncertainties and random factors exist in agricultural commodity market. Therefore, it is difficult to forecast the agricultural commodity price. In this study, the time series data of agricultural commodity price indexes were granulated by triangular fuzzy information granulation theory. Then the MEA was applied to seek the optimal parameters $c$ and $g$ to establish the hybrid MEA-SVM model. Finally, the trained MEA-SVM model was used to predict the fluctuation range and change trend of agricultural commodity price index in the future. The empirical results are as follows.

The fuzzy information granulation method can granulate the time series data of the original agricultural commodity 
price index and use the fuzzy information particles Low, $R$, and $U p$ to represent the lowest, average, and highest values of agricultural commodity price fluctuation.

The MEA can seek the global optimal parameters of SVM model in a wider range without having to traverse all the parameter points, which improves the calculation speed and prediction accuracy.

The MEA-SVM regression model based on fuzzy information granulation is more accurate and effective in predicting the fluctuation range and variation trend of agricultural commodity price index, which indicates that the model has certain advantages in nonlinear simulation, adaptive learning, and processing of incomplete complex information.

The MEA-SVM model can be generalized to predict the price fluctuations of other commodity and help to provide some reference for the time series data prediction in other fields. Nonetheless, the MEA-SVM model based on fuzzy information granulation still has some deficiencies, such as underestimation and the long-term prediction error being larger. In future studies, the penalty parameter $c$ and kernel parameter $g$ of SVM model can be further optimized through other intelligence algorithms to improve the prediction accuracy and stability.

\section{Data Availability}

Our research data comes from the Food and Agriculture Organization of the United Nations (FAO). The research data is authoritative and reliable. The FAO food price index is a measure of the monthly change in international prices of a basket of food commodities. It consists of the average of six commodity group price indexes (Food Price Index, Cereal Price Index, Vegetable Oil Price Index, Meat Price Index, Dairy Price Index, and Sugar Price Index). At the same time, the research data is public and can be accessed and obtained through the url http://www.fao.org/home/en/. Research data can be accessed and downloaded by anyone at any time.

\section{Conflicts of Interest}

The authors declare no conflicts of interest.

\section{Acknowledgments}

This paper performance was supported by Wonkwang University in 2018.

\section{References}

[1] D. Yang and Z. Liu, "Does farmer economic organization and agricultural specialization improve rural income? Evidence from China," Economic Modelling, vol. 29, no. 3, pp. 990-993, 2012.

[2] S. Yao, Y. Guo, and X. Huo, "An empirical analysis of the effects of china's land conversion program on farmers' income growth and labor transfer," Journal of Environmental Management, vol. 45 , no. 3, pp. 502-512, 2010.

[3] L.-J. Chen, C. Ye, S.-W. Hu, V. Wang, and J. Wen, "The effect of a target zone on the stabilization of agricultural prices and farmers' nominal income," Journal of Agricultural and Resource Economics, vol. 38, no. 1, pp. 34-47, 2013.

[4] S. Sriboonchitta, H. T. Nguyen, A. Wiboonpongse, and J. Liu, "Modeling volatility and dependency of agricultural price and production indices of Thailand: Static versus time-varying copulas," International Journal of Approximate Reasoning, vol. 54, no. 6, pp. 793-808, 2013.

[5] G.-Q. Li, S.-W. Xu, Z.-M. Li, Y.-G. Sun, and X.-X. Dong, "Using Quantile Regression Approach to Analyze Price Movements of Agricultural Products in China," Journal of Integrative Agriculture, vol. 11, no. 4, pp. 674-683, 2012.

[6] A. M. Kizito and J. Staatz, "A partial equilibrium approach to estimating the potential payoffs of providing improved agricultural market information in the form of price forecasts," Cahiers Agricultures, vol. 23, no. 4-5, pp. 325-335, 2014.

[7] M. K. Adjemian and A. Smith, "Using USDA forecasts to estimate the price flexibility of demand for agricultural commodities," American Journal of Agricultural Economics, vol. 94, no. 4, pp. 978-995, 2012.

[8] A. M. McKenzie, H. L. Goodwin, and R. I. Carreira, "Alternative Model Selection Using Forecast Error Variance Decompositions in Wholesale Chicken Markets," Journal of Agricultural and Applied Economics, vol. 41, no. 01, pp. 227-240, 2009.

[9] W. Huang, Z. Huang, M. Matei, and T. Wang, "Price volatility forecast for agricultural commodity futures: The role of high frequency data," Romanian Journal of Economic Forecasting, vol. 15, no. 4, pp. 83-103, 2012.

[10] G. Martín-Rodríguez and J. J. Cáceres-Hernández, "Forecasting pseudo-periodic seasonal patterns in agricultural prices," Agricultural Economics (United Kingdom), vol. 43, no. 5, pp. 531-544, 2012.

[11] V. Jadhav, B. V. Chinnappa Reddy, and G. M. Gaddi, "Application of ARIMA model for forecasting agricultural prices," Journal of Agricultural Science and Technology, vol. 19, no. 5, pp. 981-992, 2017.

[12] B. S. Yashavanth, K. N. Singh, A. K. Paul, and R. K. Paul, "Forecasting prices of coffee seeds using Vector Autoregressive Time Series Model," Indian Journal of Agricultural Sciences, vol. 87, no. 6, pp. 754-758, 2017.

[13] X. Su, Y. Wang, S. Duan, and J. Ma, "Detecting chaos from agricultural product price time series," Entropy, vol. 16, no. 12, pp. 6415-6433, 2014.

[14] T. Xiong, C. Li, and Y. Bao, "Seasonal forecasting of agricultural commodity price using a hybrid STL and ELM method: Evidence from the vegetable market in China," Neurocomputing, vol. 275, pp. 2831-2844, 2018.

[15] C. A. O. Pinheiro and V. de Senna, "Multivariate analysis and neural networks application to price forecasting in the Brazilian agricultural market," Ciência Rural, vol. 47, no. 1, 2017.

[16] Y. Wang, X. Su, and S. Guo, "The optimal confidence intervals for agricultural products' price forecasts based on hierarchical historical errors," Entropy, vol. 18, no. 12, article no. 439, 2016.

[17] T. Xiong, C. Li, Y. Bao, Z. Hu, and L. Zhang, "A combination method for interval forecasting of agricultural commodity futures prices," Knowledge-Based Systems, vol. 77, pp. 92-102, 2015.

[18] X. Kong, Y. Sun, R. Su, and X. Shi, "Real-time eutrophication status evaluation of coastal waters using support vector machine with grid search algorithm," Marine Pollution Bulletin, vol. 119, no. 1, pp. 307-319, 2017. 
[19] Y. Yao, H. Cui, Y. Liu, L. Li, L. Zhang, and X. Chen, "PMSVM: An Optimized Support Vector Machine Classification Algorithm Based on PCA and Multilevel Grid Search Methods," Mathematical Problems in Engineering, vol. 2015, Article ID 320186, 15 pages, 2015.

[20] H. Zhang, L. Chen, Y. Qu, G. Zhao, and Z. Guo, "Support vector regression based on grid-search method for short-term wind power forecasting," Journal of Applied Mathematics, vol. 2014, 2014.

[21] J. Wang, H. Du, X. Yao, and Z. Hu, "Using classification structure pharmacokinetic relationship (SCPR) method to predict drug bioavailability based on grid-search support vector machine," Analytica Chimica Acta, vol. 601, no. 2, pp. 156-163, 2007.

[22] X. Xu, D. Niu, L. Zhang, Y. Wang, and K. Wang, "Ice cover prediction of a power grid transmission line based on two-stage data processing and adaptive support vector machine optimized by genetic tabu search," Energies, vol. 10, no. 11, 2017.

[23] S. Dai, D. Niu, and Y. Han, "Forecasting of power grid investment in china based on support vector machine optimized by differential evolution algorithm and grey wolf optimization algorithm," Applied Sciences, vol. 8, no. 4, p. 636, 2018.

[24] D. Liu, D. X. Niu, H. Wang, and L. L. Fan, "Short-term wind speed forecasting using wavelet transform and support vector machines optimized by genetic algorithm," Journal of Renewable Energy, vol. 62, pp. 592-597, 2014.

[25] C. Sun, Y. Sun, and L. Wei, "Mind-evolution-based machine learning and applications," in Proceedings of the IEEE Int. Conf. on Intelligent Engineering Systems (INES'98), pp. 355-359, 1998.

[26] C. Sun, X. Zhou, and W. Wang, "Mind evolutionary computation and applications," Journal of Communication and Computer, vol. no. 1, pp. 13-21, 2004.

[27] X. Zhou and C. Sun, "Pareto-MEC and its convergence analysis," Jisuanji Gongcheng/ Computer Engineering, vol. 33, pp. 233-236, 2007.

[28] W. Wang, R. Tang, C. Li, P. Liu, and L. Luo, "A BP neural network model optimized by Mind Evolutionary Algorithm for predicting the ocean wave heights," Ocean Engineering, vol. 162, pp. 98-107, 2018.

[29] L. Xu, X. Du, and B. Wang, "Short-Term Traffic Flow Prediction Model of Wavelet Neural Network Based on Mind Evolutionary Algorithm," International Journal of Pattern Recognition and Artificial Intelligence, vol. 32, no. 12, p. 1850041, 2018.

[30] H. Liu, H. Tian, X. Liang, and Y. Li, "New wind speed forecasting approaches using fast ensemble empirical model decomposition, genetic algorithm, Mind Evolutionary Algorithm and Artificial Neural Networks," Journal of Renewable Energy, vol. 83, pp. 1066-1075, 2015.

[31] L. A. Zadeh, "Toward a theory of fuzzy information granulation and its centrality in human reasoning and fuzzy logic," Fuzzy Sets and Systems, vol. 90, no. 2, pp. 111-127, 1997.

[32] S. Yin, Y. Jiang, Y. Tian, and O. Kaynak, "A data-driven fuzzy information granulation approach for freight volume forecasting," IEEE Transactions on Industrial Electronics, vol. 64, no. 2, pp. 1447-1456, 2017.

[33] J. Guo, Z. Liu, W. Huang, Y. Wei, and J. Cao, "Short-term traffic flow prediction using fuzzy information granulation approach under different time intervals," IET Intelligent Transport Systems, vol. 12, no. 2, pp. 143-150, 2018.

[34] C. Cortes and V. Vapnik, "Support-vector networks," Machine Learning, vol. 20, no. 3, pp. 273-297, 1995.
[35] R. Noori, A. R. Karbassi, A. Moghaddamnia et al., "Assessment of input variables determination on the SVM model performance using PCA, Gamma test, and forward selection techniques for monthly stream flow prediction," Journal of Hydrology, vol. 401, no. 3-4, pp. 177-189, 2011.

[36] M. G. De Giorgi, S. Campilongo, A. Ficarella, and P. M. Congedo, "Comparison between wind power prediction models based on wavelet decomposition with least-squares support vector machine (LS-SVM) and artificial neural network (ANN)," Energies, vol. 7, no. 8, pp. 5251-5272, 2014.

[37] T. Zhou, F. Wang, and Z. Yang, "Comparative analysis of ANN and SVM models combined with wavelet preprocess for groundwater depth prediction," Water (Switzerland), vol. 9, no. 10, 2017.

[38] G. Yan, G. Xie, Y. Qiu, and Z. Chen, "MEA Based Nonlinearity Correction Algorithm for the VCO of LFMCW Radar Level Gauge," in Rough Sets, Fuzzy Sets, Data Mining, and Granular Computing, vol. 3641 of Lecture Notes in Computer Science, pp. 461-470, Springer Berlin Heidelberg, Berlin, Heidelberg, 2005.

[39] G. Li and W.-H. Li, "Face occlusion recognition based on MEBML," Jilin Daxue Xuebao (Gongxueban)/Journal of Jilin University (Engineering and Technology Edition), vol. 44, no. 5, pp. 1410-1416, 2014. 


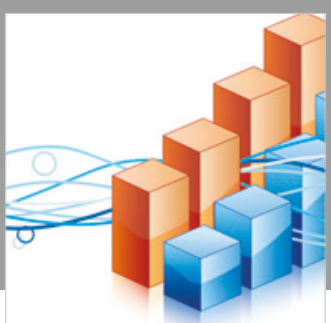

Advances in

Operations Research

\section{-n-m}
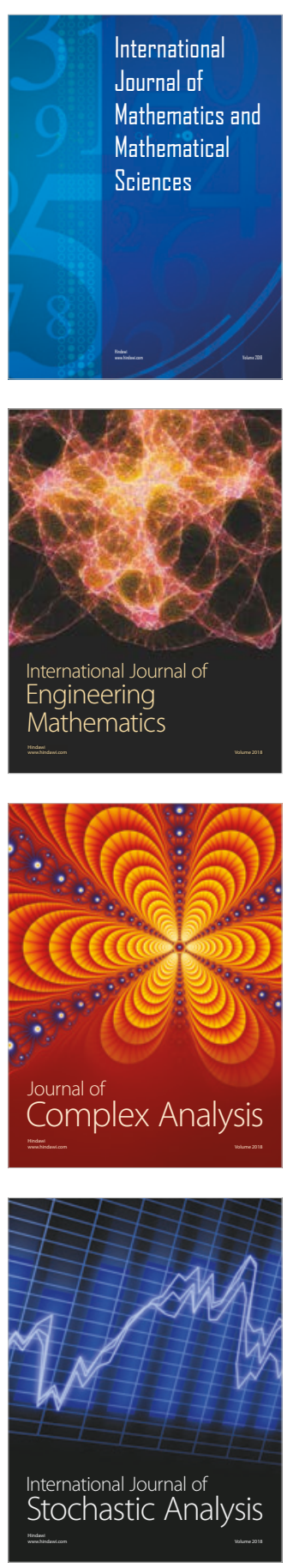
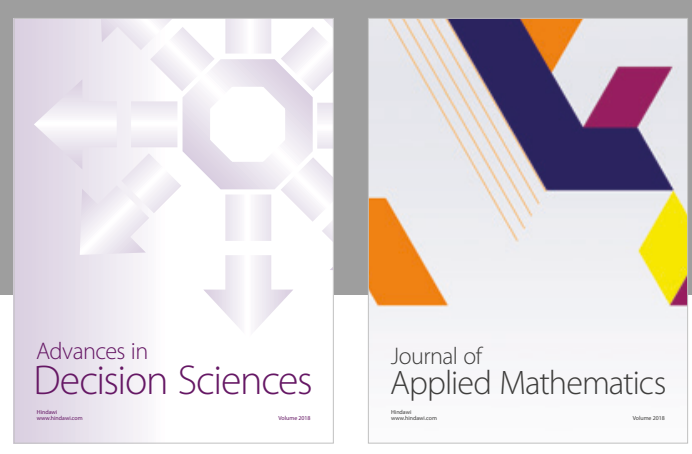

Journal of

Applied Mathematics
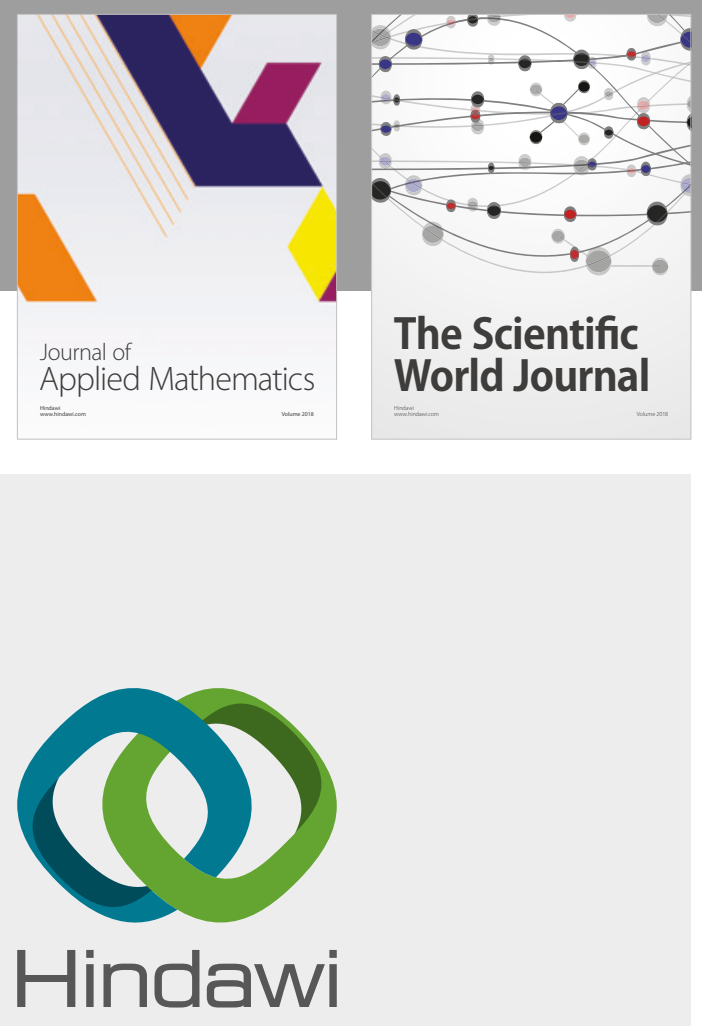

Submit your manuscripts at

www.hindawi.com

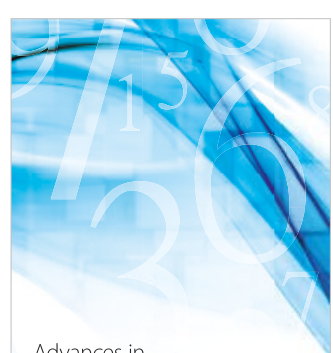

Advances in
Numerical Analysis
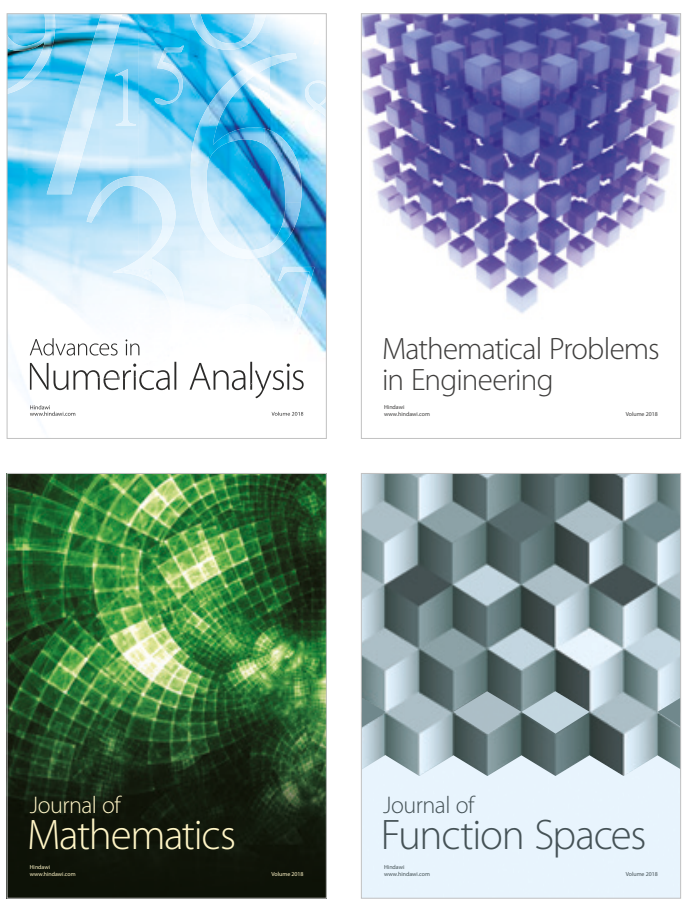

Mathematical Problems in Engineering

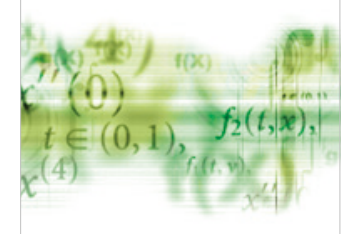

International Journal of

Differential Equations

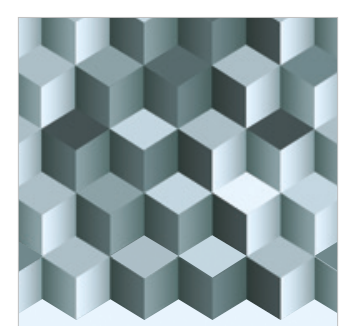

Journal of

Function Spaces

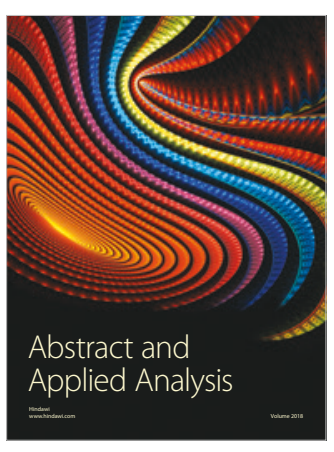

The Scientific

World Journal

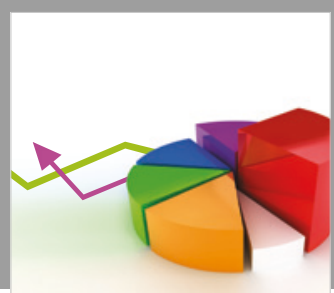

Journal of

Probability and Statistics
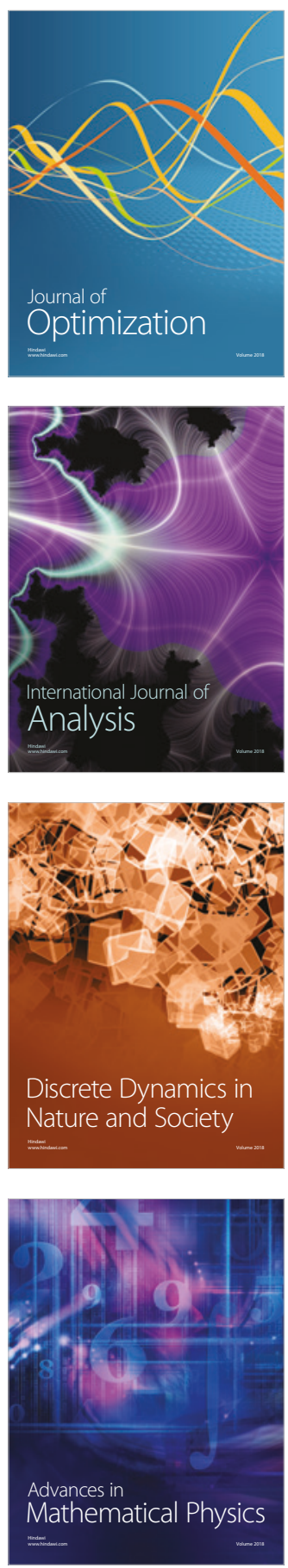